\title{
Gamificação de um Sistema de Juiz Online para Motivar Alunos em Disciplina de Programação Introdutória
}

\author{
Ralph B. S. Ribeiro, David B. F. Oliveira, Leandro S. G. Carvalho, Elaine H. T. de \\ Oliveira
}

\author{
${ }^{1}$ Instituto de Computação - Universidade Federal do Amazonas (UFAM) \\ Av. General Rodrigo Octávio, 6200 - Coroado I - CEP 69077-000 - Manaus - AM - Brasil \\ \{rbsr, david, galvao, elaine\} aicomp.ufam.edu.br
}

\begin{abstract}
Resumo. Este artigo apresenta um sistema de juiz online gamificado desenvolvido com o objetivo de motivar e engajar estudantes iniciantes no aprendizado de programação e reduzir os altos índices de reprovação em disciplinas introdutórias de programação. O sistema foi aplicado em onze turmas de diferentes cursos de engenharia e ciências exatas da Universidade Federal do Amazonas ao longo de um periodo letivo. Por meio de análises quantitativas, verificou-se um aumento no desempenho e na taxa de aprovação nas turmas que utilizaram o juiz online gamificado em relação às turmas do ano anterior, que utilizaram o sistema sem gamificação.
\end{abstract}

\begin{abstract}
This paper presents a gamified online judge system that aims to engage and motivate beginner students in programming learning, in addition to minimize high levels of fail rate in disciplines of introduction to programming. Eleven classes of different courses of engineering and exact sciences from Federal University of Amazonas used this system over a semester. By means of quantitative analyzes, we noticed that there was an increase in performance and approval rate for classes that applied the gamified online judge if compared to classes of previous year, with the non-gamified online judge.
\end{abstract}

\section{Introdução}

O ensino de programação é fundamental para cursos de graduação em computação e constitui a matriz curricular de diversos cursos das áreas de exatas, humanas e biológicas [Bosse e Gerosa 2015]. Apesar da grande importância, as disciplinas introdutórias de programação apresentam altos índices de reprovação e desistência [Bosse e Gerosa 2015] [Chaves et al. 2013].

Aprender a programar não é uma tarefa fácil, principalmente para calouros, em fase de adaptação à vida universitária. Os estudantes precisam ter uma base em lógicamatemática e dedicar tempo para resolver exercícios. Além disso, estudantes que não são de cursos de computação, ou seja, estudantes de alguns cursos de engenharia e ciências exatas, não costumam se sentir motivados a cursar essa disciplina, já que resolver problemas usando programação não é o foco do perfil de egresso desses cursos.

Por exemplo, na Universidade Federal do Amazonas (UFAM), a disciplina de Introdução à Programação de Computadores (IPC) é ministrada para 17 cursos de graduação em engenharia e de ciências exatas. Em 2016, a taxa de aprovação foi de apenas $43,3 \%$ e a taxa de desistência foi de $19,7 \%$ entre os 635 estudantes matriculados. 
VII Congresso Brasileiro de Informática na Educação (CBIE 2018)

Anais do XXIX Simpósio Brasileiro de Informática na Educação (SBIE 2018)

Uma metodologia muito discutida atualmente para aumentar a motivação dos estudantes é o uso de softwares e ferramentas gamificadas. Segundo Kapp (2012), a gamificação (do inglês "gamification") pode ser definida como "a utilização de mecânica, estética e pensamento baseados em jogos para engajar pessoas, motivar a ação, promover a aprendizagem e resolver problemas".

Para que os alunos estejam motivados na prática diária de resolução de problemas, o processo de aprendizagem precisa de uma experiência interessante sob o ponto de vista do aluno. Nesse sentido, o uso de gamificação pode amenizar esse problema devido à predisposição que os jovens têm pelo ato de jogar [Campos, Gardiman e Madeira 2015].

Portanto, o presente estudo busca avaliar o impacto de um sistema de juiz online gamificado sobre o desempenho do estudante. Para tal fim, foram formuladas duas questões de pesquisa que conduzem este estudo:

- Q1: Há influência da gamificação na nota do estudante em IPC?

- Q2: Há influência dos elementos de gamificação sobre a nota final em IPC?

Com o intuito de investigar essas questões, utilizamos um ambiente de correção automática de códigos (ou juiz online) chamado CodeBench ${ }^{1}$ [Carvalho, Oliveira e Gadelha 2016] e adicionamos técnicas de gamificação para promover o engajamento e motivar os alunos nas atividades de programação.

O artigo está organizado da seguinte forma: na Seção 2 apresenta-se a contextualização do trabalho com o referencial teórico a respeito do uso da gamificação na educação e sobre sistemas de juízes online. A Seção 3 descreve a ferramenta proposta neste trabalho. O projeto experimental e a metodologia de avaliação do sistema são descritos na Seção 4; e os resultados são discutidos na Seção 5. Por fim, a Seção 6 contém a conclusão e os trabalhos futuros.

\section{Contextualização}

Nesta seção, aborda-se o uso de gamificação na educação para melhoria da aprendizagem e sistemas de juízes online, com ênfase no sistema CodeBench.

\subsection{Gamificação na Educação}

A gamificação corresponde ao uso de mecanismos de jogos, tais como competição, desafio, iteração social e recompensa, em contextos não relacionados a jogos [Kapp 2012]. Assim, o uso de ferramentas gamificadas implica em empregar as mesmas estratégias, elementos e métodos utilizados em jogos para resolver problemas que não estão associados a jogos, comuns no mundo real.

Segundo Peixoto et al. (2015), técnicas de gamificação em softwares educacionais estão cada vez mais sendo utilizadas para apoiar a aprendizagem. Mas qual a vantagem de se usar gamificação na educação? Fardo (2013) argumenta que um dos benefícios é disponibilizar aos alunos um sistema no qual eles consigam visualizar o impacto de suas ações e sentir que estão aprendendo, no decorrer da disciplina. Assim, da mesma forma que acontece nos jogos, fica mais fácil entender que atingir pequenos objetivos contribui para alcançar um objetivo maior. Dessa maneira, para criarmos uma experiência de

\footnotetext{
${ }^{1} \mathrm{http}: / /$ codebench.icomp.ufam.edu.br/
} 
VII Congresso Brasileiro de Informática na Educação (CBIE 2018)

Anais do XXIX Simpósio Brasileiro de Informática na Educação (SBIE 2018)

aprendizagem divertida e eficaz, precisamos conseguir transmitir no sistema o "pensamento de game", ou seja, necessitamos combinar os elementos de jogos com uma experiência de aprendizagem adequada (Figura 1) [Alves 2014].

Assim, como argumenta Kaap (2012), a gamificação pode ser utilizada para promover a aprendizagem, e cada elemento de jogo deve estar conectado entre si, pois os elementos, sozinhos, não são suficientes para transformar uma aula tradicional em uma experiência motivadora e engajadora, embora cada elemento seja importante e fundamental no processo geral da gamificação.

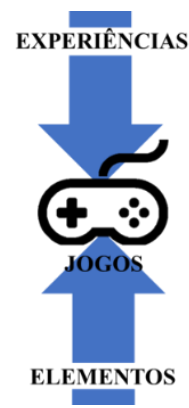

Figura 1. Pensamento de game. Fonte: Adaptado de Alves (2014).

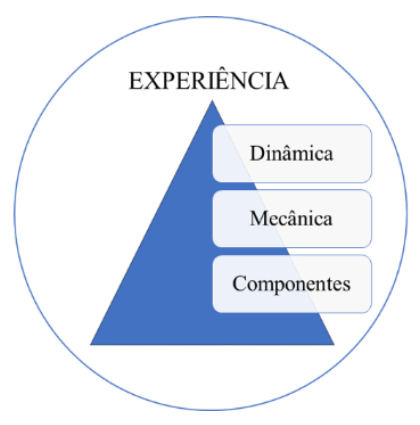

Figura 2. Pirâmide representando a hierarquia de elementos em um projeto de gamificação. Fonte: Adaptado de Werbach e Hunter (2012).

Werbach e Hunter (2012) mostram que a relação entre a mecânica, a dinâmica e os componentes, expressa na Figura 2, "é essencial para o sucesso de um projeto com o uso de gamificação". Ainda segundo eles, a dinâmica é "constituída por elementos responsáveis por atribuir coerência e padrões regulares à experiência". É ela que determina as regras e os elementos mais conceituais, como: restrições, emoções, narrativa, progressão e relacionamento.

Já na mecânica dos jogos estão os elementos que promovem a "ação", ou seja, é como se eles fossem a "engrenagem" do sistema que faz a gamificação funcionar no decorrer da execução. Dentre eles, podemos citar: desafios, sorte, cooperação e competição, feedback, aquisição de recursos, recompensas, transações, turnos e estados de vitória. Por fim, na base da pirâmide, os componentes representam a essência da dinâmica e da mecânica do jogo, são eles: realizações, avatares, medalhas (badges), coleções, combates, desbloqueio de conteúdo, placares (rankings), níveis, pontos, exploração, entre outros.

Este estudo busca dotar um juiz online com elementos de gamificação, para gerar uma experiência de aprendizagem motivadora em IPC, como será visto a seguir.

\subsection{Sistema de Juiz Online}

Juízes online "são plataformas web cujo propósito é compilar, executar e avaliar programas submetidos por usuários" [Wasik et al. 2018].

$\mathrm{Na}$ UFAM, utiliza-se o sistema de juiz online chamado CodeBench para dar suporte a professores e estudantes em disciplinas iniciais de programação. Por meio desse sistema, professores e tutores das disciplinas podem disponibilizar exercícios de programação, listas e até mesmo provas para seus alunos, que por sua vez podem desenvolver soluções em uma IDE (Integrated Development Environment) acoplada ao 
VII Congresso Brasileiro de Informática na Educação (CBIE 2018)

Anais do XXIX Simpósio Brasileiro de Informática na Educação (SBIE 2018)

próprio sistema. Assim que o estudante submete o código de solução para um dado exercício, o sistema compara a string de saída do programa do estudante com a string cadastrada como correta, informando instantaneamente o resultado.

Após a adoção desse sistema como ferramenta de apoio a uma metodologia de ensino híbrido de programação, houve um aumento no índice de aprovações dos alunos de IPC [Carvalho, Oliveira e Gadelha 2016]. Porém, esse sistema, como dito anteriormente, apenas dá um suporte ao professor da disciplina automatizando vários processos, como: correção manual de exercícios, atribuição de notas, entre outros, mas não foca em motivar ou incentivar o aluno a resolver as questões propostas ou a participar ativamente da disciplina.

\section{O sistema Gamificado}

O juiz online foi gamificado utilizando-se a metodologia de design instrumental ADDIE [Branch 2009] unificada com o modelo ISD (Instructional Systematic Design) de Dick e Carey (2001). Essa abordagem unificada do matriciamento entre as principais etapas desses dois modelos foi proposta por Wangenheim e Wangenheim (2012). Seguindo a metodologia deles, nas etapas iniciais de desenvolvimento, foi concebida a mecânica de gamificação e foram definidas a meta do jogo, suas regras e a forma de interação do sistema, como mostrado na Tabela 1.

Tabela 1. Concepção do juiz online gamificado

\begin{tabular}{|c|c|}
\hline $\begin{array}{r}\text { Concepção da } \\
\text { gamificação }\end{array}$ & Juiz online gamificado \\
\hline Meta & $\begin{array}{l}\text { Motivar o aluno a resolver exercícios de programação disponibilizados no } \\
\text { juiz online }\end{array}$ \\
\hline Tipo de mídia & Digital e online \\
\hline Contexto & Utilizado ao longo da disciplina \\
\hline Interação & Individual \\
\hline Narrativa & $\begin{array}{l}\text { A narrativa se passa em um mundo de fantasia medieval onde, ao final da } \\
\text { jornada, os personagens (estudantes de uma mesma turma) devem enfrentar } \\
\text { um monstro (Quimera) e libertar o país Midgard de sua dominação }\end{array}$ \\
\hline Descrição & $\begin{array}{l}\text { O estudante escolhe seu avatar entre várias opções disponíveis. À medida } \\
\text { que vai resolvendo exercícios, ele anda pelo mapa até Quimera, ganhando } \\
\text { pontos de força e armas. Quanto maior a força e melhor a arma do avatar, } \\
\text { mais pontos de vida consegue tirar do monstro ao enfrentá-lo }\end{array}$ \\
\hline Resultados & $\begin{array}{l}\text { É necessário que uma porcentagem da turma chegue até o final do mapa, } \\
\text { onde está Quimera, para matá-la. Essa porcentagem é definida pelo } \\
\text { professor no momento de criação da turma e pode ser alterada a qualquer } \\
\text { momento, desde o início até o final das aulas. Apesar da interação ser } \\
\text { individual, esse objetivo coletivo foi inserido para que a dinâmica da } \\
\text { gamificação não se tornasse excessivamente competitiva. A morte da } \\
\text { Quimera define o "estado vencedor" do jogo }\end{array}$ \\
\hline Feedback & $\begin{array}{l}\text { Durante a utilização do juiz online, os alunos recebem feedback do seu } \\
\text { desempenho, de acordo com a posição, armas e a força do seu personagem }\end{array}$ \\
\hline
\end{tabular}


VII Congresso Brasileiro de Informática na Educação (CBIE 2018)

Anais do XXIX Simpósio Brasileiro de Informática na Educação (SBIE 2018)

- Força: 1 a $n$ pontos de força.

- Posição no mapa: 1 a $n$ casas.

Caso os estudantes obtenham nota máxima em uma lista de exercícios, o seu avatar ganha uma medalha (badge), representada por uma arma mais potente. Quanto melhor a arma usada pelo avatar, mais pontos de vida ele consegue tirar da Quimera.

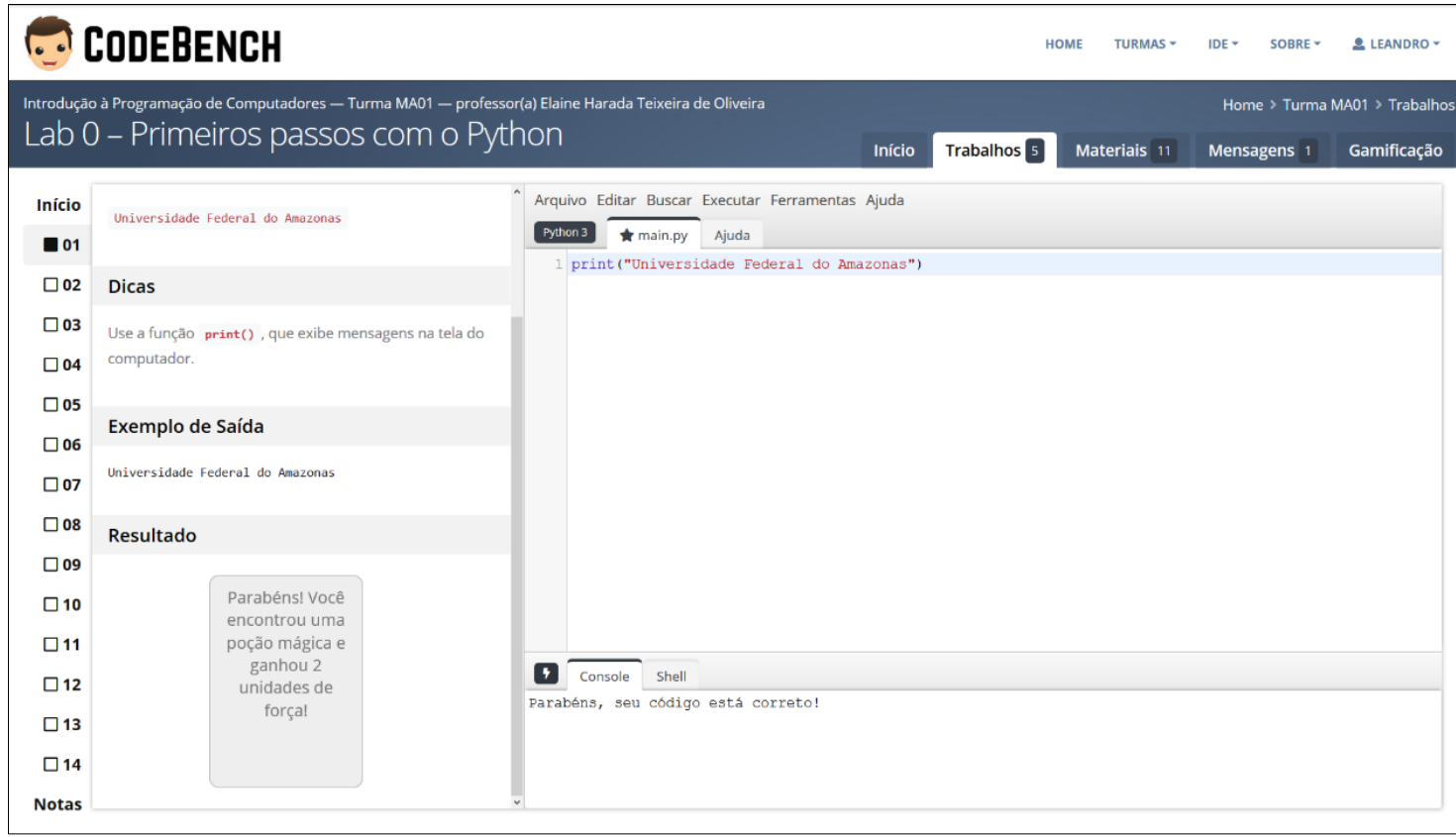

Figura 3. Captura de tela de uma questão submetida com sucesso.

A Figura 4 mostra a captura de tela do mapa e a Figura 5 o ranking dos jogadores.

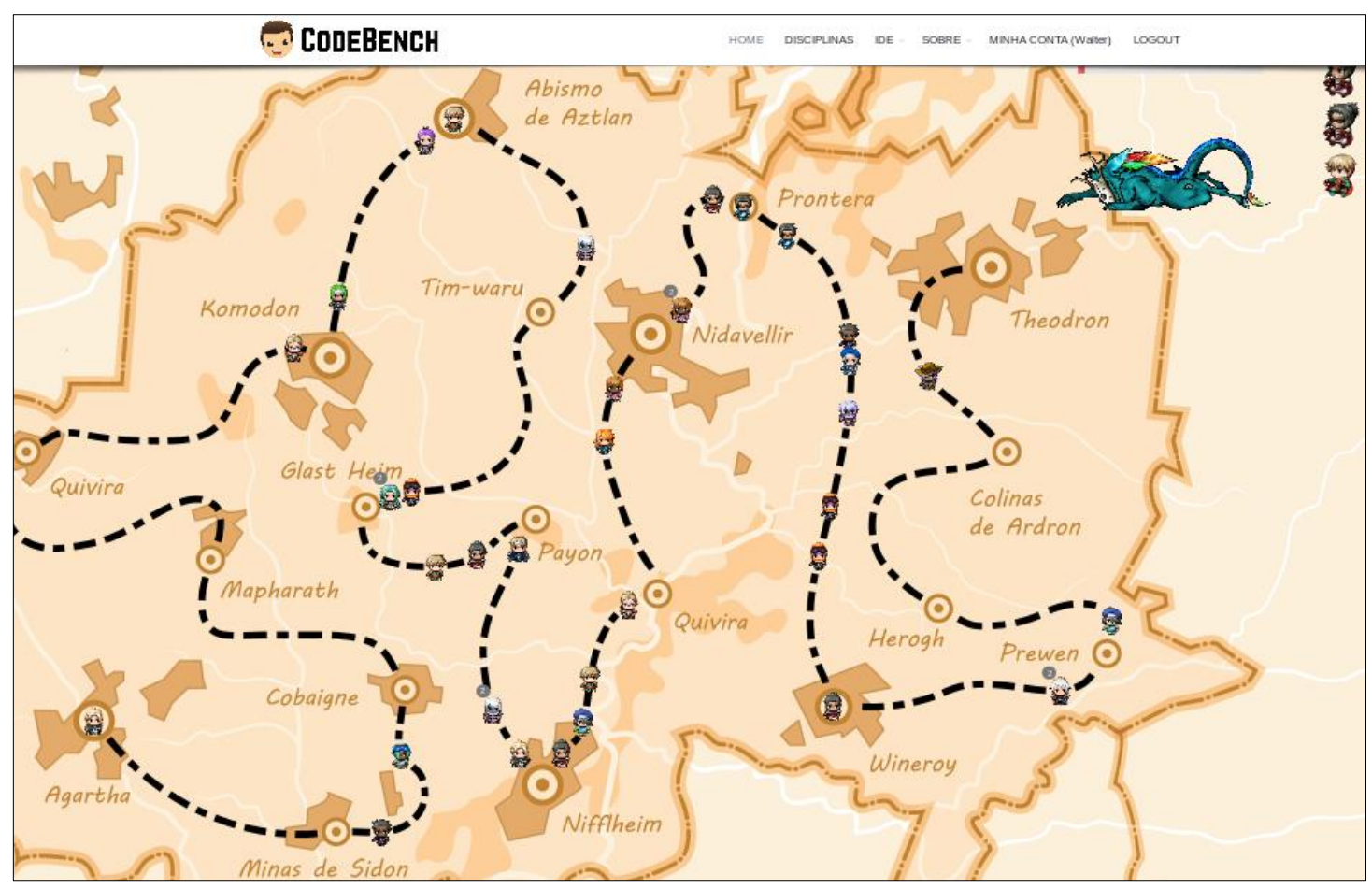

Figura 4. Captura de tela do mapa com a posição dos avatares. 
VII Congresso Brasileiro de Informática na Educação (CBIE 2018)

Anais do XXIX Simpósio Brasileiro de Informática na Educação (SBIE 2018)

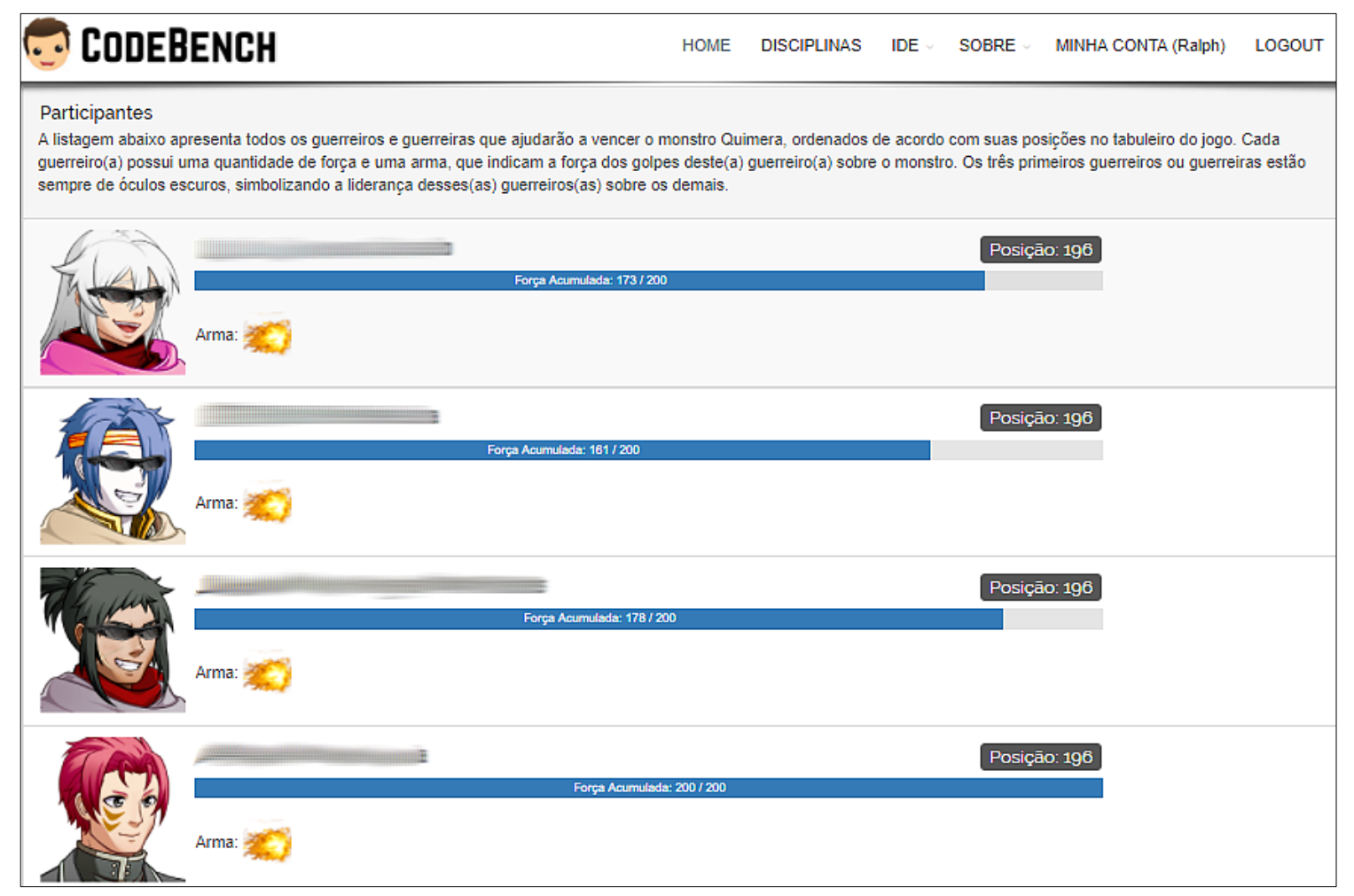

Figura 5. Ranking dos personagens no sistema gamificado.

A mecânica e componentes também utilizam elementos como: desafios, competição, badges, feedback, recompensas, estado de vitória, combate, ranking, pontos e bens virtuais.

\section{Projeto Experimental}

Foi realizado um quase-experimento para mensurar o desempenho do estudante ao utilizar o CodeBench. Para tanto, o sistema gamificado foi aplicado na UFAM, durante o primeiro semestre letivo de 2017, na disciplina Introdução à Programação de Computadores (IPC), em onze turmas da área de ciências exatas e engenharias.

As mesmas turmas ofertadas no período letivo anterior correspondente $(2016 / 1)$ foram utilizadas como "grupo de controle" para efeito de comparação, nas quais os alunos usaram o sistema CodeBench sem gamificação.

Foram coletados dados de 460 alunos do grupo experimental (2017/1) e 493 do grupo controle (2016/1), totalizando 953 alunos, a partir de duas fontes:

- Dados de Suporte 1 (DS01) - Uma planilha de dados, extraída a partir do sistema de registro acadêmico, contendo informações dos estudantes matriculados em IPC em todos os cursos que ofertaram a disciplina em 2016/1 e 2017/1.

- Dados de Suporte 2 (DS02) - Um log, extraído do juiz online, que contém as ações individuais dos alunos no sistema gamificado durante a disciplina.

Durante a fase de planejamento, foram formuladas três hipóteses a partir das perguntas de pesquisa. Para a Q1, foi formulada a hipótese nula H01:

- H01: Não há diferença entre as notas dos estudantes das turmas do grupo experimental e as notas das turmas do grupo de controle. 
VII Congresso Brasileiro de Informática na Educação (CBIE 2018)

Anais do XXIX Simpósio Brasileiro de Informática na Educação (SBIE 2018)

Com relação à Q2, foram formuladas as hipóteses nulas H02 e H03:

- H02: Não há correlação entre os pontos de força adquiridos pelos estudantes no sistema gamificado e a nota final do aluno na disciplina de IPC.

- H03: Não há correlação entre a posição final do estudante no mapa do sistema gamificado e a nota final do aluno na disciplina de IPC.

O sistema gamificado foi avaliado quantitativamente, considerando-se a nota final dos estudantes em ambos os grupos (experimental e de controle) e a correlação entre dois elementos da gamificação (força e posição final) e essa mesma nota. Também foi verificada a taxa de aprovação dos estudantes não desistentes na disciplina em ambos os grupos. Os resultados são apresentados na Seção 5.

\section{Resultados}

Nesta seção, são apresentados os resultados quantitativos obtidos a partir das hipóteses levantadas durante o projeto de experimento.

\subsection{Desempenho em IPC}

Para testar a H01, comparamos as notas finais dos estudantes matriculados em 2017/1 (grupo experimental) com as notas finais dos estudantes de 2016/1 (controle), utilizando o teste não-paramétrico de Mann-Whitney. O valor $p$ obtido foi de 0,069 , de modo que não podemos afirmar que há diferença significativa entre o desempenho dos alunos que utilizaram o sistema gamificado e o desempenho dos alunos da turma de controle.

Por outro lado, quando se exclui os estudantes desistentes (reprovados por falta ou que trancaram a disciplina.), o valor $p$ obtido é 6,61e-09, rejeitando-se assim a H01. Ou seja, podemos afirmar que há diferença nas notas dos estudantes não desistentes, quando comparamos as turmas do grupo experimental e de controle.

Os estudantes podem desistir da disciplina por diversos fatores que não estão ligados necessariamente ao seu desempenho ou à metodologia adotada:

1. A disciplina de IPC é ministrada para estudantes de $1^{\circ}$ ou $3^{\circ}$ período. Nesse momento, é comum que alguns deles verifiquem que não têm afinidade com o curso escolhido, o que pode levar à evasão do curso, independente da disciplina.

2. Estudantes de cursos que ofertam disciplinas de programação como atividademeio tendem a priorizar disciplinas ligadas ao curso, que são pré-requisitos para disciplinas que julgam ser mais importantes;

3. Outras disciplinas com alto índice de reprovação, como Cálculo I e Álgebra Linear I [Silva et. al 2016] são ministradas concomitantemente com IPC, fazendo o estudante "disputar" tempo de estudo com essas disciplinas.

Por isso, optou-se por excluir da análise os alunos que trancaram ou reprovaram por frequência em IPC. Dessa forma, ameniza-se o viés causado por esses motivos, e consegue-se mensurar com mais exatidão o desempenho real do aluno na disciplina.

De uma forma geral, a Figura 6(a) mostra as taxas de aprovação, reprovação por nota e desistência de todos os alunos matriculados em IPC. Apesar de não podermos afirmar estatisticamente que houve diferença nas notas quando comparamos todos os alunos, o gráfico mostra uma melhoria das taxas de aprovação e uma redução da taxa de reprovação por nota na turma experimental em relação à turma de controle. A Figura 6(a) 
VII Congresso Brasileiro de Informática na Educação (CBIE 2018)

Anais do XXIX Simpósio Brasileiro de Informática na Educação (SBIE 2018)

também mostra que houve um aumento na taxa de desistência (reprovação por falta e trancamentos). Os possíveis motivos que podem levar um aluno a desistir da disciplina foram relatados previamente. As causas desse aumento nas desistências serão investigadas em trabalhos futuros.

Na Figura 6(b), a caixa à esquerda representa a distribuição das notas de 351 dos 493 alunos de 2016/1 (grupo controle), e a caixa à direita representa a distribuição das notas de 294 dos 460 alunos de 2017/1 (grupo experimental).

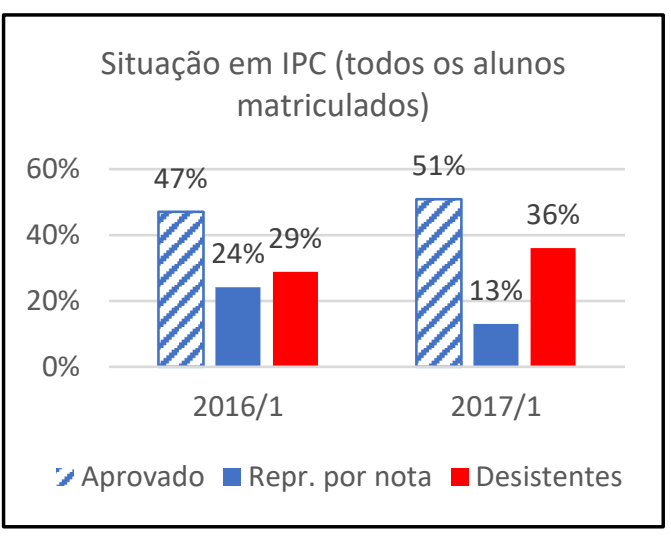

(a)

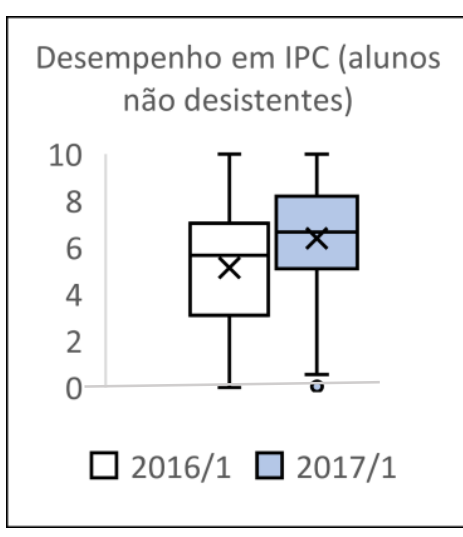

(b)

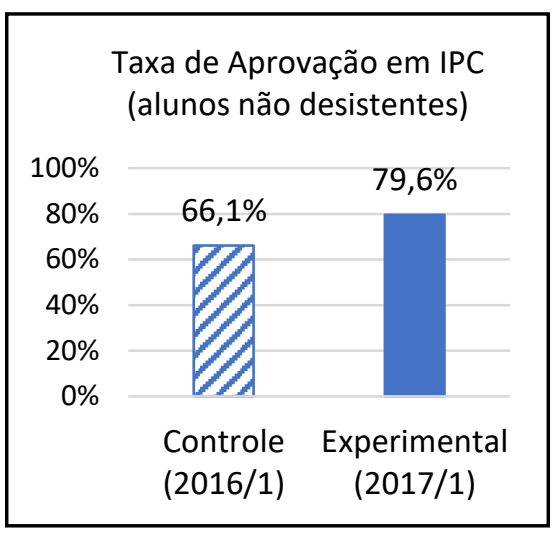

(c)

Figura 6. (a) Comparação das taxas de aprovação, reprovação por nota e desistência entre as turmas de 2016/1 e 2017/1, considerando todos os alunos matriculados. (b) Comparação da distribuição de notas entre os estudantes não desistentes das turmas de 2016/1 e 2017/1. (c) Comparação das taxas de aprovação dos estudantes não desistentes entre as turmas 2016/1 e 2017/1.

O diagrama de caixa indica uma melhora nas médias das notas das turmas do grupo experimental em relação às do grupo controle, de 5,13 para 6,40. Outra melhoria evidente também ocorreu na mediana, que saltou de 5,67 em 2016/1 para 6,67 em 2017/1. Esses resultados são indícios quantitativos de que a metodologia com o sistema de juiz online gamificado contribuiu para a melhoria do desempenho dos estudantes que não trancaram a disciplina ou não reprovaram por frequência.

A Figura 6(c) apresenta a taxa de aprovação em IPC em 2016/1 e 2017/1, considerando todos os alunos matriculados não desistentes. Houve uma melhoria desse índice para as turmas que utilizaram o sistema de juiz online gamificado em relação ao grupo de controle, como consequência do aumento de desempenho.

Carvalho, Oliveira e Gadelha (2016) apresentaram uma metodologia que une aulas tradicionais com um sistema de juiz online, que provocou um aumento na taxa de aprovação em IPC. Dando continuidade a esse trabalho, o presente estudo mostra, por meio dos testes quantitativos que foi possível ampliar esse ganho com o uso da gamificação.

\subsection{Elementos de Gamificação $\times$ Desempenho}

Ao final da disciplina, todas as 11 turmas conseguiram derrotar a Quimera. Além disso, dos 234 alunos aprovados, 80 (34,2\%) conseguiram chegar à última posição do mapa. Mesmo depois de o monstro ter sido derrotado, os demais alunos que não alcançaram a Quimera ainda podiam resolver exercícios e avançar casas no mapa. 
VII Congresso Brasileiro de Informática na Educação (CBIE 2018)

Anais do XXIX Simpósio Brasileiro de Informática na Educação (SBIE 2018)

As hipóteses H02 e H03 foram verificadas por meio da correlação de Spearman. Os resultados na Tabela 2 indicam que houve uma correlação forte ${ }^{2}\left(\boldsymbol{r}_{\boldsymbol{s}}>0,7\right)$ entre os pontos de força conquistados pelos estudantes no sistema gamificado e seu desempenho na disciplina. O mesmo ocorre nos testes de correlação entre posição dos jogadores no mapa da gamificação e seu desempenho em IPC. Como se encontrou um valor $p \ll 0,05$, rejeitaram-se as hipóteses $\mathrm{H} 02$ e H03 de que esses valores de correlação são iguais a zero.

Tabela 2. Coeficiente de correlação de Spearman $\left(r_{s}\right)$ entre os pontos e a posição no mapa $\times$ notas dos estudantes em IPC

\begin{tabular}{rcc}
\hline & Pontos de Força $\times$ Notas & Posição no Mapa $\times$ Notas \\
\hline Nível de significância & $p$-valor $=2,2 \mathrm{e}-16$ & $p$-valor $=2,2 \mathrm{e}-16$ \\
\hline Coeficiente de correlação & $r_{s}=0,74$ & $r_{s}=0,74$ \\
\hline
\end{tabular}

Então, conclui-se que os elementos da gamificação contidos no juiz online têm forte associação com as notas dos estudantes. Ou seja, o elemento "sorte" inserido na mecânica da gamificação não distorce a equidade que se espera de que os estudantes com maior pontuação no jogo apresentem também maior desempenho em termos de notas.

\subsection{Ameaças à Validade}

Apesar dos bons resultados obtidos nos testes quantitativos, temos que considerar alguns fatores que ameaçam sua validade. Um deles é que as turmas foram ministradas por professores distintos entre os diferentes cursos. Assim, para amenizar esse possível viés, os materiais didáticos e as atividades foram os mesmos nos dois grupos (experimental e de controle). Além disso, pode ter havido diferença nas dificuldades das questões das avaliações entre os anos de 2016 e 2017. Contudo, esse efeito foi amenizado designandose diferentes professores para elaborar avaliações distintas.

\section{Conclusão e Trabalhos Futuros}

$\mathrm{O}$ ato de programar e resolver problemas exige a mobilização de diversas habilidades cognitivas do aprendiz. Grande parte dos estudantes que ingressam em cursos superiores vem do ensino médio com uma base lógico-matemática deficiente e isso acaba influenciando na aprendizagem de algoritmos. Além disso, muitos estudantes que não têm familiaridade com programação tendem a não se interessar por esse tipo de disciplina. Essa falta de interesse gera desmotivação e faz o aluno desistir da disciplina ante a pequenas dificuldades. A gamificação pode ajudá-los nesse sentido, pois torna a aprendizagem divertida e desafiadora. Além disso, os estudantes sentem que estão evoluindo na disciplina quando avançam no sistema gamificado. Esse feedback, junto com a colaboração e a competição, são poderosas ferramentas de engajamento estudantil.

Neste artigo apresentamos o desenvolvimento e utilização de um juiz online gamificado com a fim de motivar alunos na aprendizagem de programação. Mostramos, por meio de análises quantitativas, a evolução no desempenho dos estudantes, e consequentemente, o aumento na taxa de aprovação comparado a turmas anteriores.

Como um trabalho futuro, além daqueles mencionados ao longo do texto, esperase apresentar os resultados das análises qualitativas que mediram, por meio de um

\footnotetext{
${ }^{2}$ Segundo Dancey e Reidy (2006): 0,1 $<\left|\boldsymbol{r}_{s}\right| \leq 0,3$ (fraco); 0,4 $<\left|\boldsymbol{r}_{s}\right| \leq 0,6$ (moderado); e 0,7 $<$ $\left|r_{s}\right| \leq 1,0$ (forte).
} 
VII Congresso Brasileiro de Informática na Educação (CBIE 2018)

Anais do XXIX Simpósio Brasileiro de Informática na Educação (SBIE 2018)

questionário, o sentimento dos alunos em relação à motivação, aprendizagem e a utilização do sistema CodeBench gamificado.

\section{Referências}

Alves, F. (2015). Gamification: como criar experiências de aprendizagem engajadoras, 2ed. DVS editora.

Bosse, Y.; Gerosa, M. A. (2015). Reprovações e Trancamentos nas Disciplinas de Introdução à Programação da Universidade de São Paulo: Um Estudo Preliminar. In XXIII Workshop sobre Educação em Computação. Recife.

Branch, R. M. (2009). Instructional design: The ADDIE approach (Vol. 722). Springer Science \& Business Media.

Campos A.; Gardiman R.; Madeira C. (2015). Aplicação da Gamificação na Disciplina de Empreendedorismo. In XXIII Workshop sobre Educação em Computação. Recife.

Chaves, J. O. M.; Castro, A. F.; Lima, R. W.; Lima, M. V. A.; Ferreira, K. H. (2013). Integrando Moodle e Juízes Online no Apoio a Atividades de Programação. In Anais do Simpósio Brasileiro de Informática na Educação (Vol. 24, No. 1, p. 244).

Dancey, C.; Reidy, J. (2013). Estatística Sem Matemática para Psicologia, 5ed. Porto Alegre, Artmed.

Dick, W.; Carey, L.; Carey, J. O. (2001). The Systematic Design of Instruction, 7ed. Allyn \& Bacon.

Fardo, M. L. (2013). A gamificação como estratégia pedagógica: estudos de elementos dos "games" aplicados em processos de ensino e aprendizagem. Dissertação de mestrado em educação, Universidade de Caxias do Sul, Caxias do Sul, RS.

Carvalho, L. S. G.; Oliveira, D. B. F.; Gadelha, B. F. (2016). Juiz online como ferramenta de apoio a uma metodologia de ensino híbrido em programação. In Simpósio Brasileiro de Informática na Educação-SBIE) (Vol. 27, No. 1, p. 140).

Kapp, K. (2012). The gamification of learning and instruction: game-based methods and strategies for training and education. John Wiley \& Sons.

Peixoto, M. M; Silva, C; Gonçalves, E; Vilena, J. (2015). Um Mapeamento Sistemático de Gamificação em Software Educativo no Contexto da Comunidade Brasileira de Informática na Educação. Anais do XXI Workshop de Informática na Escola.

Silva, A. C.; Correa, C. S.; Coelho, D. A.; Silva Neto, D. T.; Ferraz, L.; Xavier, M. M.; Reis, R. S.; Rocha, F. A.; Santos, P. A. (2016). Análise dos Índices de Reprovação nas Disciplinas de Cálculo I e AVGA do Curso de Engenharia Elétrica do Instituto Federal da Bahia de Vitória da Conquista. INTERTECH 2016.

von Wangenheim, C. G.; von Wangenheim, A. (2012). Ensinando computação com jogos. Bookess Editora, Florianópolis, SC, Brasil.

Wasik, S.; Antczak, M.; Laskowski, A.; Sternal, T. (2018). A Survey on Online Judge Systems and Their Applications. ACM Computing Surveys, 51(1), 3.

Werbach, K.; Hunter, D. (2012). For the win: How game thinking can revolutionize your business. Wharton Digital Press. 